RAIRO Operations Research

RAIRO Oper. Res. 39 (2005) 275-293

DOI: $10.1051 /$ ro:2006006

\title{
RECHERCHE À VOISINAGE VARIABLE DE GRAPHES EXTRÉMAUX 13. À PROPOS DE LA MAILLE*
}

\author{
Mustapha Aouchiche ${ }^{1}$ et Pierre Hansen ${ }^{2}$
}

Résumé. Le système AutoGraphiX (AGX1 et AGX2) permet, parmi d'autres fonctions, la génération automatique de conjectures en théorie des graphes et, dans une version plus récente, la preuve automatique de conjectures simples. Afin d'illustrer ces fonctions et le type de résultats obtenus, nous étudions systématiquement ici des conjectures obtenues par ce système et de la forme $\underline{b}_{n} \leq g \oplus i \leq \bar{b}_{n}$ où $g$ désigne la maille (ou longueur du plus petit cycle) du graphe $G=(V, E), i$ un autre invariant choisi parmi le nombre de stabilité, le rayon, le diamètre, le degré minimum, moyen ou maximum, $\underline{b}_{n}$ et $\bar{b}_{n}$ des fonctions de l'ordre $n=|V|$ de $G$ les meilleures possibles, enfin $\oplus$ correspond à une des opérations ,,$+- \times, / .48$ telles conjectures sont obtenues : les plus simples sont démontrées automatiquement et les autres à la main. De plus 12 autres conjectures ouvertes et non encore étudiées sont soumises aux lecteurs.

Mots Clés. Graphe, invariant, conjecture, AGX, maille.

\begin{abstract}
The AutoGraphiX system (AGX1 et AGX2) allows, among other functions, automated generation of conjectures in graph theory and, in its most recent version, automated proof of simple conjectures. To illustrate these functions and the type of results obtained, we study systematically in this paper, conjectures of the form $\underline{b}_{n} \leq g \oplus i \leq \bar{b}_{n}$ where $g$ denotes the girth (or length of the smallest cycle) of a graph
\end{abstract}

Reçu le 20 janvier 2005. Accepté le 9 décembre 2005.

* Cet article est le treizième de la série "Variable Neighborhood Search for Extremal Graphs" publiée à partir de 1998 (voir bibliographie). La recherche présentée a bénéficié du support de la Chaire HEC en Exploitation de Données et de la subvention CRSNG No. 105574-1998.

1 Département de mathématiques et génie industriel, École Polytechnique de Montréal, Qc, Canada; Mustapha.Aouchiche@gerad.ca

2 GERAD et Service de l'enseignement des méthodes quantitatives de gestion HEC Montréal, Qc, Canada; Pierre.Hansen@gerad.ca

(c) EDP Sciences 2006 
$G=(V, E), i$ another invariant among independence number, radius,iameter, minimum, average or maximum degree, $\underline{b}_{n}$ and $\bar{b}_{n}$ best possible functions of the order $n$ of $G$, and $\oplus$ denotes one of the four operations,,$+- \times, / .48$ such conjectures are obtained : the easiest ones are proved automatically and the others by hand. Moreover 12 open and unstudied conjectures are submitted to the readers.

\section{INTRODUCTION}

Comme dans de nombreux autres domaines mathématiques, l'ordinateur joue un rôle croissant en théorie des graphes. L'aspect algorithmique de cette théorie a été exploré de longue date et les applications en sont nombreuses. Plus récemment, l'ordinateur a été utilisé pour faire progresser la théorie elle-même, c'est-à-dire obtenir des conjectures, des réfutations ou des preuves. Une revue des travaux, déjà nombreux, en ce domaine est faite dans [15]. Une discussion plus approfondie de la génération automatique ou assistée de conjectures en théorie des graphes (en particulier à l'aide des trois systèmes opérationnels GRAPH [11], Graffiti [12] et AutoGraphiX [7]) est présentée dans [16].

Le système AutoGraphiX a été développé au GERAD, Montréal à partir de 1997 par Gilles Caporossi, Pierre Hansen et leurs collaborateurs. Ses buts principaux sont les suivants :

(a) obtenir un graphe satisfaisant des contraintes données;

(b) obtenir un graphe minimisant (ou maximisant) un invariant éventuellement sous contraintes (ou une formule impliquant plusieurs invariants, qui est elle-même un invariant c'est-à-dire une fonction bien définie ne dépendant pas de la numérotation des sommets ou des arêtes du graphe);

(c) réfuter une conjecture (ou la réparer, ou la renforcer par l'addition de termes supplémentaires);

(d) obtenir une conjecture (par exemple sous la forme d'une relation algébrique entre invariants ou une description de la structure des graphes extrémaux obtenus);

(e) prouver une conjecture simple (dans la deuxième version, AGX2) ou bien suggérer une idée de preuve, ou montrer qu'une telle idée ne peut suffire à elle seule à prouver une conjecture (dans la première version, AGX1).

L'idée de base est de considérer ces problèmes comme des problèmes d'optimisation combinatoire paramétrique sur une famille infinie de graphes $G=(V, E)$, dont toutefois seuls les membres d'ordre $n=|V|$ et/ou de taille $m=|E|$ modérés feront l'objet de calculs.

Soit $i$ un invariant graphique, $\mathcal{G}_{n}$ (ou $\mathcal{G}_{n, m}$ ) la famille de tous les graphes d'ordre $n$ (ou d'ordre $n$ et de taille $m$ ). AutoGraphiX utilise une approche générique de l'optimisation d'un invariant $i$, c'est-à-dire une méthode heuristique de résolution du problème suivant, pour tout $i$

$$
\begin{aligned}
& \operatorname{Min} / \operatorname{Max} i \text { ou } \operatorname{Min} / \operatorname{Max} i \text {. } \\
& G \in \mathcal{G}_{n} \quad G \in \mathcal{G}_{n, m}
\end{aligned}
$$


Pour ce faire la métaheuristique de Recherche à Voisinage Variable (RVV) [19,20] fournit le cadre général. Cette métaheuristique (ou cadre général pour construire des heuristiques) exploite systématiquement l'idée de changement de voisinage, tant dans une phase de descente vers un optimum local que dans une phase de sortie de la vallée correspondante.

Dans une première version, AGX1 [7,8], AutoGraphiX exploite la RVV en phase de descente par l'application d'une série de transformations élémentaires définissant autant de voisinages du graphe courant : rotation, retrait ou addition d'une arête, détour (remplacement d'une arête par une chaîne de longueur 2), racourcis (l'inverse de l'opération précédente) et ainsi de suite. Dans une version plus récente, AGX2 [1], toutes les transformations possibles portant sur un sous-graphe de 2 , puis 3 , puis 4 sommets sont prises en compte. On atteint ainsi un optimum local, correspondant à un graphe $G^{*}$. Le graphe $G^{*}$ est alors perturbé de manière croissante par l'addition ou la suppression d'une, deux, trois arêtes ou plus. Après chaque perturbation la phase de descente est reitérée jusqu'à atteindre un nouvel optimum local correspondant à un graphe $G^{\prime}$. On est alors dans une des trois situations suivantes : $(i)$ le graphe $G^{\prime} \equiv G^{*}$; on procède à une nouvelle perturbation, plus importante; $(i i)$ le graphe $G^{\prime} \neq G^{*}$; et la valeur $i\left(G^{\prime}\right)$ est moins bonne que $i\left(G^{*}\right)$; on procède comme dans le cas $(i)$ ci-dessus; $(i i i)$ le graphe $G^{\prime} \neq G^{*}$; et la valeur $i\left(G^{\prime}\right)$ est meilleure que la valeur $i\left(G^{*}\right)$; la recherche est alors recentrée en $G^{\prime}$. Elle se poursuit jusqu'à ce qu'une condition d'arrêt (par exemple un temps maximum de calcul ou un nombre maximum d'itération sans amélioration) soit satisfaite.

Les graphes obtenus pour diverses valeurs de $n$ et $m$ ne sont pas nécessairement optimaux, car la résolution du problème de minimisation ou de maximisation de $i$ a été faite à l'aide d'une heuristique. L'expérience montre cependant que les graphes optimaux sont très souvent obtenus, du moins pour $n \leq 10$. Pour de plus grandes valeurs, diverses améliorations de l'heuristique peuvent être faites (par exemple, le choix du graphe initial $G$ peut se faire parmi des familles de graphes qui sont souvent extrémales, ou encore une série de telles familles peuvent être testées ; une correction automatique ou interactive peut être faite sur des graphes qui diffèrent fortement des autres, etc.).

La famille de graphes extrémaux obtenue est exploitée de trois manières pour obtenir des conjectures [16] :

(a) une méthode numérique, basée sur les mathématiques de l'analyse en composantes principales, qui fournit une base de relations affines entre invariants ;

(b) une méthode géométrique qui consiste à déterminer la fermeture convexe de l'ensemble des graphes extrémaux obtenus considérés comme des points dans l'espace des invariants;

(c) une méthode algébrique, qui consiste à reconnaître la (ou les) famille(s) de graphes extrémaux obtenus et à substituer les expressions des invariants apparaissant dans $i$ en fonction de $n$, puis à simplifier. 
Les approches (a) et (c) seront illustrées dans la suite. D'autres applications à des problèmes de théorie pure des graphes (notamment de la théorie spectrale) sont présentées dans $[3,7,8,10,18]$ et à des problèmes de chimie mathématique dans $[5,6,14,17]$.

Afin de tester systématiquement la version AGX2 du système AutoGraphiX, une famille générale de relations a été récemment considérée [2]. Ces relations sont de la forme :

$$
\underline{b}_{n} \leq i_{1} \oplus i_{2} \leq \bar{b}_{n}
$$

où $i_{1}$ et $i_{2}$ sont deux invariants de $G, \oplus$ désigne une des oprérations,,$+- \times$, et $/, \underline{b}_{n}$ et $\bar{b}_{n}$ sont des fonctions de l'ordre $n$ de $G$ les meilleures possibles, au sens fort, c'est-à-dire telles que pour toute valeur de $n$, il existe un graphe $G^{\prime}$ (resp. $\left.G^{\prime \prime}\right)$ pour lequel la borne inférieure (resp. supérieure) est atteinte.

Les relations de la forme (1) généralisent les formules du type Nordhaus-Gaddum [21], souvent étudiées, de deux manières : $(i)$ on considère deux invariants $i_{1}$ et $i_{2}$ plutôt que le même invariant dans $G$ et le graphe complémentaire $\bar{G}$ (deux sommets sont adjacents dans $\bar{G}$ si et seulement si ils ne le sont pas dans $G$ ); $(i i)$ on considère en plus des opérations + et $\times$ les opérations - et $/$. Les formules obtenues ainsi ont plusieurs avantages : elles sont souvent simples (on retrouve par exemple le théorème de Chung [9] $\bar{l} \leq \alpha$ où $\bar{l}$ désigne la distance moyenne entre paires de sommets de $G$ et $\alpha$ le nombre d'indépendence) et précises (on améliore, par exemple, la conjecture $\bar{l} \cdot \delta \leq n$ de Graffiti [24] en la conjecture $\bar{l} \cdot \delta \leq n-1$ qui est la meilleure possible et a été prouvée par le premier auteur ainsi que par Smith [22]). Enfin, la difficulté des preuves est variable, sans qu'elles ne soient en général trop compliquées.

Le présent article a deux buts :

(i) illustrer les méthodes de découverte automatique de conjectures ainsi que de preuve automatique de conjectures simples d'AGX2 ;

(ii) permettre une évaluation des résultats obtenus en considérant un invariant donné et plusieurs autres, ainsi que toutes les opérations citées.

Pour ce faire, nous étudions systématiquement les conjectures de la forme (1) où $i_{1}$ est la maille $g$ de $G$ et $i_{2}$ un autre invariant. Les sections 2 à 4 suivantes correspondent aux cas où $i_{2}$ est le nombre de stabilité, puis le rayon et le diamètre et enfin le degré maximum, moyen et minimum. Les valeurs extrêmes des invariants pour les graphes connus avec au moins 3 sommets utilisées dans les preuves sont présentées dans le tableau 1.

Les conjectures les plus simples sont prouvées automatiquement par AGX2 en vérifiant que l'intersection des ensembles de graphes extrémaux (pour la minimisation ou la maximisation selon le cas) pour $i_{1}$ et $i_{2}$ est non-vide. Si c'est le cas il suffit de remplacer dans la formule correspondante les expressions de $i_{1}$ et $i_{2}$ en fonction de $n$.

Cette méthode de preuve automatique peut être étendue, en considérant des secondes plus grandes ou plus petites valeurs, ou en exploitant une base de relations connues plus large que celle du tableau 1. Cela ne s'est cependant pas avéré nécessaire pour les problèmes considérés dans cet article. 
TABLEAU 1. Les bornes et les graphes extrémaux pour les invariants.

\begin{tabular}{|c|c|c|c|c|}
\hline & \multicolumn{2}{|c|}{ Borne inférieure } & \multicolumn{2}{c|}{ Borne supérieure } \\
\hline Invariant & Graphes extrémaux & Formule & Graphes extrémaux & Formule \\
\hline$g$ & Graphes avec triangle & 3 & Cycles & $n$ \\
\hline$\alpha$ & Graphes complets & 1 & Etoiles & $n-1$ \\
\hline$r$ & $\begin{array}{c}\text { Graphes avec un } \\
\text { sommet dominant }\end{array}$ & 1 & Chemin, cycles, ... & $\left\lfloor\frac{n}{2}\right\rfloor$ \\
\hline$D$ & Graphes complets & 1 & Chemins & $n-1$ \\
\hline$\Delta$ & Chemins et cycles & 2 & $\begin{array}{c}\text { Graphes avec } \\
\text { sommet dominant }\end{array}$ & $n-1$ \\
\hline $\bar{d}$ & Arbres & $2-\frac{2}{n}$ & Graphes complets & $n-1$ \\
\hline$\delta$ & $\begin{array}{c}\text { Graphes avec un } \\
\text { sommet pendant }\end{array}$ & 1 & Graphes complets & $n-1$ \\
\hline
\end{tabular}

Certaines conjectures étaient déjà connues ou évidentes; les preuves des autres sont fournies ci-dessous. Enfin, pour laisser le lecteur juger de la facilité ou difficulté des résultats obtenus, nous présentons dans la section 5 une série de 12 conjectures (concernant la maille et d'autres invariants) sans avoir essayé de les prouver. Deux de ces conjectures portent seulement sur la structure des graphes extrémaux car elles impliquent l'index $\lambda_{1}$ qui est difficile à manipuler. Une autre difficulté apparaît dans quatre conjectures impliquant l'indice de Randić : la suppression d'une arête peut le faire augmenter ou diminuer. De brèves conclusions sont données dans la section 6 .

\section{LA MAILle ET LE NOMBRE DE STABiLité}

Un ensemble de sommets $A \subset V$ de $G$ est stable si et seulement si les sommets de $A$ sont deux à deux non adjacents. Le nombre de stabilité (ou nombre d'indépendence) $\alpha$ de $G$ est la cardinalité du plus grand ensemble stable de $G$.

Les résultats (simples) obtenus et prouvés automatiquement lors de la comparaison de la maille avec le nombre de stabilité sont :

$$
\alpha+g \geq 4 \quad \text { et } \quad \alpha \cdot g \geq 3,
$$

avec égalité, dans les deux cas, si et seulement si le graphe est complet. Ces relations découlent directement des bornes inférieures sur $\alpha$ et $g$ du tableau 1 et de ce que l'intersection de la famille de graphes avec triangles et des graphes complets est constituée des graphes complets.

Les autres conjectures issues de la comparaison de $\alpha$ et de $g$ sont aussi aisées à obtenir automatiquement. Leur preuve n'est pas non plus difficile. 
Proposition 2.1. Soit $G$ un graphe connexe avec $n \geq 3$ sommets et $m \geq n$ arêtes. Alors :

$$
\alpha+g \leq n+\lfloor n / 2\rfloor .
$$

De plus la borne est atteinte si et seulement si $G$ est un cycle.

Preuve. Le sous-graphe induit par le plus petit cycle de $G$ contient au plus $\lfloor g / 2\rfloor$ sommets du stable maximum, d'où

$$
\begin{aligned}
& \alpha \leq\lfloor g / 2\rfloor+n-g \\
& \text { et } \\
& \alpha+g \leq\lfloor g / 2\rfloor+n \leq\lfloor n / 2\rfloor+n \text {. }
\end{aligned}
$$

La borne est atteinte si et seulement si $g=n$, c'est-à-dire si $G$ est un cycle.

La relation (1) est obtenue automatiquement par AGX2 à l'aide de la méthode algébrique; des graphes extrémaux sont identifiés comme étant des cycles $C_{n}$, les relations $\alpha\left(C_{n}\right)=\lfloor n / 2\rfloor$ et $g\left(C_{n}\right)=n$ sont extraites de la base de relations et sommées.

Proposition 2.2. Soit $G$ un graphe connexe avec $n \geq 3$ sommets et $m \geq n$ arêtes. Alors :

$$
-\lceil n / 2\rceil \leq \alpha-g \leq n-5
$$

De plus la borne inférieure est atteinte si et seulement si $G$ est un cycle et la borne supérieure si et seulement si $G$ est un triangle et $n-3$ arêtes pendantes incidentes à deux sommets au plus de ce triangle.

Preuve. Pour la borne inférieure,

$$
\alpha \geq\lfloor g / 2\rfloor \Longrightarrow \alpha-g \geq\lfloor g / 2\rfloor-g=-\lceil g / 2\rceil \geq-\lceil n / 2\rceil .
$$

d'où la borne. Elle est atteinte si et seulement si $g=n$ c'est-à-dire si $G$ est un cycle.

Pour la borne supérieure, comme $G$ a au moins un cycle, $\alpha \leq n-2$ et $g \geq 3$, d'où le résultat. Cette borne est atteinte si et seulement si ces deux conditions sont des égalités, c'est-à-dire si $G$ est un triangle et $n-3$ arêtes pendantes incidentes à deux sommets au plus de ce triangle.

Les bornes inférieures et supérieures de la relation (3) sont obtenues automatiques par AGX2 à l'aide de la méthode numérique. Pour la borne supérieure, le système considère les valeurs de $\alpha-g$ pour $n \leq 3,4, \ldots, 15$ et cherche une courbe passant par ces points de la forme $a_{1} n+a_{2} n^{2}+a_{3} / n+a_{4}$ où $a_{1}, a_{2}, a_{3}$ et $a_{4}$ sont des paramètres, ce qui donne $n-5$. Pour la borne inférieure, cette première étape ne donnant pas de résultat, AGX2 considère séparément les valeurs de $n$ paires et impaires, ce qui donne les solutions :

$$
\begin{aligned}
-\frac{n}{2} & \leq \alpha-g & & \text { pour } n \text { pair } \\
-\frac{n+1}{2} & \leq \alpha-g & & \text { pour } n \text { impair }
\end{aligned}
$$

et le résultat. 
Proposition 2.3. Soit $G$ un graphe connexe avec $n \geq 3$ sommets et $m \geq n$ arêtes. Alors :

$$
\alpha \cdot g \leq\left\lfloor\frac{n^{2}}{2}\right\rfloor .
$$

De plus la borne est atteinte si et seulement si $G$ est un cycle pour $n$ pair et un cycle sur $n-1$ sommets avec un sommet pendant si $n$ est impair.

Preuve. La relation (2) implique

$$
\alpha \cdot g \leq\lfloor g / 2\rfloor g+n g-g^{2} .
$$

Si $g$ est pair, le membre de droite de (4) est égal à

$$
n g-g^{2} / 2
$$

et sa dérivée par rapport à $g$ est

$$
n-g \geq 0 .
$$

Si $g$ est impair, le membre de droite de (4) devient égal à

$$
n g-\frac{g^{2}}{2}-\frac{g}{2}
$$

et sa dérivée par rapport à $g$ est

$$
n-g-\frac{1}{2}
$$

qui est non négative sauf si $g=n$ (et $n$ est impair).

La borne est maximum pour $n=g$ si $n$ est pair et pour $g=n-1$ si $n$ est impair, car alors $\alpha=\frac{n+1}{2}$ et $g=n-1$ d'où $\alpha g=\frac{n^{2}-1}{2}$, ce qui est plus grand que $\alpha=\frac{n-1}{2}, g=n$ et $\alpha g=\frac{n^{2}-n}{2}$. Dans les deux cas la borne est atteinte.

Proposition 2.4. Soit $G$ un graphe connexe avec $n \geq 3$ sommets et $m \geq n$ arêtes. Alors :

$$
\frac{1}{3} \leq \frac{\alpha}{g} \leq \frac{n-2}{3} .
$$

La borne inférieure est atteinte si et seulement si $G$ est un graphe complet et la borne supérieure si et seulement si $G$ est un triangle et $n-3$ arêtes pendantes incidentes à deux sommets au plus de ce triangle.

Preuve. Pour la borne inférieure, comme $\alpha \geq\left\lfloor\frac{g}{2}\right\rfloor$, on a $\frac{\alpha}{g} \geq\left\lfloor\frac{g}{2}\right\rfloor / g$ qui est égal à $\frac{1}{2}$ si $g$ est pair et à $(g-1) / 2 g$ si $g$ est impair. Cette dernière expression est minimum et égale à $1 / 3$ pour $g=3$. Alors $\alpha=1$ et $G$ est un graphe complet.

La borne supérieure se démontre comme celle de la proposition 2.2 . 


\section{LA MAILle ET LES DISTANCES}

\subsection{LE RAYON}

La distance entre deux sommets $u$ et $v$ d'un graphe $G$, notée $d_{G}(u, v)$ est la longueur d'une plus courte chaîne les reliant. L'excentricité d'un sommet $u$ de $G$ est la plus grande distance de ce sommet à un autre sommet c'est-à-dire $\operatorname{exc}(u)=$ $\max \left\{d_{G}(u, v), v \in V\right\}$. Le rayon d'un graphe est la plus petite de ses excentricités, c'est-à-dire que $r=\min \{\operatorname{exc}(u), u \in V\}$. Le diamètre d'un graphe est la plus grande de ses excentricités, c'est-à-dire que $D=\max \{\operatorname{exc}(u), u \in V\}=$ $\max \left\{d_{G}(u, v), u, v \in V\right\}$. Lors de la recherche automatique de formules du type (1), AGX2 trouve et prouve facilement les relations suivantes :

$$
4 \leq r+g \leq\left\lfloor\frac{n}{2}\right\rfloor+n \quad \text { et } \quad 3 \leq r \cdot g \leq n \cdot\left\lfloor\frac{n}{2}\right\rfloor .
$$

Les bornes inférieures sont atteintes pour les graphes contenant un sommet dominant et au moins un triangle, et les bornes supérieures si et seulement si le graphe est un cycle.

AGX2 obtient également les conjectures prouvées dans les propositions suivantes.

Proposition 3.1. Soit $G$ un graphe connexe d'ordre $n \geq 3$, de rayon $r$ et de maille finie $g$. Alors :

$$
-\left\lceil\frac{n}{2}\right\rceil \leq r-g \leq\left\lfloor\frac{n-1}{2}\right\rfloor-3
$$

La borne inférieure est atteinte pour les cycles et la borne supérieure pour les cycles avec une corde formant un triangle, et autres graphes de rayon $r=\left\lfloor\frac{n-1}{2}\right\rfloor$ et de maille $g=3$.

Preuve. Borne inférieure : pour un graphe $G$ de rayon $r$ et de maille $g$ on a $r \geq\left\lfloor\frac{g}{2}\right\rfloor$, donc,

$$
r-g \geq\left\lfloor\frac{g}{2}\right\rfloor-g \geq-\left\lceil\frac{g}{2}\right\rceil \geq-\left\lceil\frac{n}{2}\right\rceil .
$$

D'où le résultat. La borne est atteinte si et seulement si $g=n$, c'est-à-dire $G$ est un cycle.

Borne supérieure : pour tout graphe connexe contenant au moins un cycle, on a $r \leq\left\lfloor\frac{n}{2}\right\rfloor$ et $g \geq 3$, si $n$ est impair le résultat est évident $\left(\left\lfloor\frac{n}{2}\right\rfloor=\left\lfloor\frac{n-1}{2}\right\rfloor\right)$. Donc, il nous reste à prouver le cas où $n$ est pair. Avec la condition $m \geq n, r=\left\lfloor\frac{n}{2}\right\rfloor=\frac{n}{2}$ si et seulement si $G$ est un cycle. Si $G$ est un cycle, $r-g=-\frac{n}{2}<\left\lfloor\frac{n-1}{2}\right\rfloor$. Donc nécessairement $r-g \leq\left\lfloor\frac{n-1}{2}\right\rfloor-3$.

Pour tout $n$, la borne est atteinte pour plusieurs familles de graphes avec $r=$ $\left\lfloor\frac{n-1}{2}\right\rfloor$ et $g=3$, comme :

- un cycle ou un chemin plus une arête qui forme un triangle;

- un cycle ou un chemin plus deux arêtes croisées sur 4 sommets successifs; 
- si $n$ est pair, on considère 5 sommets successifs sur un cycle ou un chemin, puis on en relie au moins deux pour former un triangle et on ajoute d'autres arêtes entre les 5 sommets sans toutefois relier le premier et le cinquième sommet.

Proposition 3.2. Soit $G$ un graphe connexe d'ordre $n \geq 3$, de rayon $r$ et de maille finie $\mathrm{g}$. Alors :

$$
\frac{1}{3} \leq \frac{r}{g} \leq \frac{1}{3}\left\lfloor\frac{n-1}{2}\right\rfloor
$$

La borne inférieure est atteinte pour les graphes avec un sommet dominant et au moins un triangle, et la borne supérieure pour les cycles avec une corde formant un triangle, et autres graphes de rayon $r=\left\lfloor\frac{n-1}{2}\right\rfloor$ et de maille $g=3$.

Preuve. Borne inférieure : comme $r \geq\left\lfloor\frac{g}{2}\right\rfloor$, on a $r / g \geq\left\lfloor\frac{g}{2}\right\rfloor / g$ qui est égal à $\frac{1}{2}$ si $g$ est pair et à $\frac{1}{2} \cdot \frac{g-1}{g}$ si $g$ est impair. Cette dernière expression est minimum et égale à $\frac{1}{3}$ pour $g=3$. Alors $r=1$ et $G$ possède un sommet dominant et un triangle.

La borne supérieure se démontre de la même façon et est atteinte pour les mêmes graphes que la borne supérieure de la proposition 3.1.

\subsection{LE DIAMÈTRE}

Lors de la comparaison automatique de la maille avec le diamètre, AGX2 prouve les résultats simples suivants :

$$
D+g \geq 4 \quad \text { et } \quad D \cdot g \geq 3,
$$

avec égalité si et seulement si le graphe est complet.

On obtient aussi, sous forme de conjectures, les propositions ci-dessous.

Proposition 3.3. Soit $G$ un graphe connexe d'ordre $n \geq 3$, de diamètre $D$ et de maille finie $\mathrm{g}$. Alors :

$$
-\left\lceil\frac{n}{2}\right\rceil \leq D-g \leq n-5
$$

La borne inférieure est atteinte pour les cycles. La borne supérieure est atteinte par des graphes ayant l'une des structures suivantes:

- un triangle et un chemin incident à un des sommets du triangle;

- un triangle et deux chemins incidents chacun à un des sommets du triangle (sans restriction sur la répartition du nombre de sommets des chemins);

- un $K_{4}-e$ (graphe complet sur 4 sommets moins une arête) et un chemin incident à un des deux sommets non-adjacents du $K_{4}-e$;

- un $K_{4}-e$ et deux chemins incidents chacun à un des deux sommets non adjacents du $K_{4}-e$ (sans restriction sur la répartition du nombre de sommets des chemins). 
Preuve. La borne inférieure est une conséquence immédiate de celle de la proposition 3.1.

Borne supérieure : avec les notations définies et les conditions exprimées dans l'énoncé, on a : $D \leq n-2$ et $g \geq 3$. Donc on a bien la borne supérieure, c'est-à-dire : $D-g \leq n-5$.

Soit $G$ un graphe pour lequel la borne est atteinte, c'est-à-dire : $D=n-2$ et $g=3$. Notons $P=u_{1} u_{2} u_{3} \cdots u_{n-1}$ le chemin réalisant le diamètre et $u_{n}$ le $n^{e}$ sommet de $G$. Le sommet $u_{n}$ est voisin d'au moins 2 sommets successifs de $P$, pour former un cycle de longueur 3 , et d'au plus 3 sommets successifs. S'il y a plus de 3 sommets ou s'ils ne sont pas successifs, alors $D<n-2$. Donc les graphes de l'énoncé sont bien les seuls graphes extrêmes pour la borne supérieure.

Proposition 3.4. Soit $G$ un graphe connexe d'ordre $n \geq 3$, de diamètre $D$ et de maille finie $\mathrm{g}$. Alors :

$$
D+g \leq\left\lfloor\frac{3 n}{2}\right\rfloor .
$$

La borne est atteinte pour les cycles si n est pair, et pour les cycles ou les cycles de longueur $n-1$ avec un sommet pendant si $n$ est impair.

Preuve. Remarquons d'abord qu'une plus courte chaîne entre deux sommets de $G$ (tel que défini dans l'énoncé) contient au plus $\lfloor g / 2\rfloor$ sommets de la maille de $G$ et au plus les $n-g$ sommets qui ne sont pas sur la maille. Donc $D \leq\lfloor g / 2\rfloor+n-g=$ $n-\lceil g / 2\rceil$. Alors $D+g \leq n+\lfloor g / 2\rfloor \leq n+\lfloor n / 2\rfloor$. Ce qui prouve la borne.

Soit $G$ un graphe pour lequel la borne est atteinte. Alors, $n+\lfloor n / 2\rfloor=D+g \leq$ $n+\lfloor g / 2\rfloor \leq n+\lfloor n / 2\rfloor$. Donc, on a $\lfloor g / 2\rfloor=\lfloor n / 2\rfloor$ et par conséquent, soit $g=n$ soit $g=n-1$.

Si $g=n$, alors nécessairement $G$ est un cycle. Si $g=n-1$, (dans ce cas $n$ est nécessairement impair) $G$ contient un cycle sur $n-1$ sommets et le $n^{e}$ sommet est relié à exactement un sommet du cycle.

Proposition 3.5. Soit $G$ un graphe connexe d'ordre $n \geq 3$, de diamètre $D$ et de maille finie $\mathrm{g}$. Alors :

$$
\frac{1}{3} \leq \frac{D}{g} \leq \frac{n-2}{3}
$$

La borne inférieure est atteinte pour les graphes complets. La borne supérieure est atteinte par des graphes ayant l'une des structures suivantes :

- un triangle et un chemin incident à un des sommets du triangle;

- un triangle et deux chemins incidents chacun à un des sommets du triangle (sans restriction sur la répartition du nombre de sommets des chemins);

- un $K_{4}-e$ (graphe complets sur 4 sommets moins une arête) et un chemin incident à un des deux sommets non-adjacents du $K_{4}-e$;

- un $K_{4}-e$ et deux chemins incidents chacun à un des deux sommets non adjacents du $K_{4}-e$ (sans restriction sur la répartition du nombre de sommets des chemins). 
Preuve. La borne inférieure est une conséquence de celle de la proposition 3.2 ; toutefois les graphes extrêmes ne sont pas les mêmes. La présente borne est atteinte si et seulement si $G$ est complet $(D=1)$.

Pour la borne supérieure, on sait que $D=n-1$ si et seulement si le graphe est un chemin. Donc si $g<\infty$, alors $D \leq n-2$, et comme $g \geq 3$, on obtient $D / g \leq(n-2) / 3$.

La caractérisation des graphes extrêmes pour la borne supérieure se fait exactement comme dans la proposition 3.3.

Proposition 3.6. Soit $G$ un graphe connexe d'ordre $n \geq 3$, de diamètre $D$ et de maille finie $g$. Alors :

$$
D \cdot g \leq\left\lfloor\frac{n^{2}}{2}\right\rfloor
$$

La borne est atteinte pour les cycles dans le cas où $n$ est pair, et pour les cycles de longueur $n-1$ avec une arête pendante si $n$ est impair.

Preuve. On a déjà noté que $D \leq n-\lceil g / 2\rceil$, donc $D \cdot g \leq(n-\lceil g / 2\rceil) \cdot g$.

Si $g$ est pair, $D \cdot g \leq n g-g^{2} / 2$ et le second membre de l'inégalité atteint son maximum $(i)$ pour $n=g$ si $n$ est pair, le graphe correspondant étant un cycle et la valeur de la borne $n^{2} / 2$, et $(i i)$ pour $g=n-1$ si $n$ est impair, le graphe correspondant étant composé d'un cycle sur $n-1$ sommets, le $n^{e}$ sommet étant voisin d'un seul sommet du cycle et la valeur de la borne supérieure étant $\left(n^{2}-1\right) / 2$.

Si $g$ est impair, $D \cdot g \leq n g-\left(g^{2}+g\right) / 2$ et le second membre de l'inégalité atteint son maximum $(i)$ pour $n=g$ si $n$ est impair et le graphe correspondant est un cycle, ou ( $i i)$ pour $g=n-1$ si $n$ est pair et le graphe correspondant est composé d'un cycle sur $n-1$ sommets et une arête pendante. Dans ce cas ( $g$ impair) la valeur de la borne supérieure est $\left(n^{2}-n\right) / 2$.

En comparant les deux bornes (selon la parité de $g$ ), il est immédiat que les graphes extrêmes correspondent au cas où $g$ est pair.

\section{LA MAILle ET LES DEGRÉS}

Le degré d'un sommet $v$ d'un graphe $G$, noté $d(v)$ est le nombre de ses voisins. Le degré maximum d'un graphe $G$, noté $\Delta$ est le maximum des degrés des sommets de $G$, c'est-à-dire que $\Delta=\max \{d(v), v \in G\}$. Le degré minimum d'un graphe $G$, noté $\delta$ est le minimum des degrés des sommets de $G$, c'est-à-dire que $\delta=$ $\min \{d(v), v \in G\}$. Le degré moyen d'un graphe $G$, noté $\bar{d}$, est le moyenne des degrés des sommets de $G$, c'est-à-dire que $\bar{d}=\frac{1}{n} \sum_{v \in G} d(v)$.

\subsection{LE DEGRÉ MAXIMUM}

Lors de la comparaison systématique de la maille et du degrés maximum, $A G X 2$ formule et prouve les résultats suivants :

$$
2-n \leq \Delta-g \leq n-4 \quad \text { et } \quad 2 / n \leq \Delta / g \leq(n-1) / 3 .
$$


Les bornes inférieures sont atteintes uniquement pour les cycles, et les bornes supérieures sont atteintes si et seulement si le graphe contient au moins un sommet dominant $(\Delta=n-1)$ et au moins $n$ arêtes.

AGX2 obtient également les conjectures prouvées dans les deux propositions suivantes.

Proposition 4.1. Soit $G$ un graphe connexe d'ordre $n \geq 4$, de degré maximum $\Delta$ et de maille finie $g$. Alors :

$$
6 \leq \Delta+g \leq n+2
$$

De plus la borne inférieure est atteinte si et seulement si le graphe est tel que $\Delta=g=3$ ou pour $C_{4}$, un cycle sur 4 sommets, et la borne supérieure pour les graphes ayant l'une des trois structures suivantes :

- un graphe avec un sommet dominant et $g=3$;

- un graphe de maille $g=4$ composé d'un sommet $v$ de degré $n-2$, un sommet $u$ qui n'est pas voisin de $v$ et adjacent à au moins 2 des $n-2$ voisins de $v$ et les voisins de $v$ forment un ensemble stable;

- un graphe de maille $g \geq 5$ composé d'un cycle de longueur $g$ et les $n-g$ sommets qui ne sont pas sur le cycle forment un ensemble stable et sont tous adjacents au même sommet du cycle.

Preuve. Pour la borne inférieure, si $\Delta=2$ et $g<\infty$ alors nécessairement $G$ est un cycle et $\Delta+g=n+2$. Dans le cas contraire, on a $\Delta \geq 3$ et $g \geq 3$, d'où la borne inférieure. Cette dernière est atteinte si et seulement si le graphe contient un triangle $(g=3)$ et est de degré maximum $\Delta=3$.

Pour la borne supérieure, considérons un graphe $G$ de maille finie $g$ et soit $v$ un sommet de $G$. Il y a deux possibilités :

- Le sommet $v$ appartient à un cycle $C$ de longueur $g$. Dans ce cas $v$ est voisin d'exactement deux sommets sur $C$ et d'au plus $n-g$ sommets de $G$ qui ne sont pas sur $C$. Ainsi $d(v) \leq n-g+2$ et donc $d(v)+g \leq n+2$.

- Le sommet $v$ n'est sur aucun cycle de longueur $g$.

Si $g=3$, il est évident que $d(v)+g \leq n+2$.

Si $g=4$, le sommet $v$ ne peut être voisin d'au moins deux sommets d'un cycle de longueur égale à la maille (sinon $G$ contiendrait un triangle), donc $d(v) \leq n-2$ et $d(v)+g \leq n+2$.

Si $g \geq 5$, le sommet $v$ est voisin d'au plus un sommet de tout cycle de longueur égale à la maille (sinon il y aurait un cycle de longueur inférieure à $g)$, donc $d(v) \leq(n-1)-(g-1)=n-g$ et $d(v)+g \leq n<n+2$.

Ainsi pour tout sommet $v$ de $G, d(v)+g \leq n+2$ et par conséquent $\Delta+g \leq n+2$. Soit maintenant $G$ un graphe tel que $\Delta+g=n+2$.

- Si $g=3$, alors $\Delta=n-1$ et $G$ contient un sommet dominant et un triangle.

- Si $g=4$, alors $\Delta=n-2$. Soit $v$ un sommet de degré $n-2$, alors les voisins de $v$ forment un ensemble stable (sinon $G$ contiendrait un triangle) et l'unique sommet non adjacent à $v$ doit avoir au moins 2 voisins communs avec $v$ pour former un cycle de longueur $g=4$. 
- Si $5 \leq g<n$, alors $\Delta=n-g+2$. On a vu plus haut que, dans ce cas, un sommet qui n'est pas sur un cycle de longueur égale à la maille est de degré au plus $n-g$. Alors le sommet $v$ de degré maximum est nécessairement sur un cycle $C$ de longueur $g$. Comme $v$ admet exactement 2 voisins sur $C$, il est nécessairement adjacent à tous les sommets qui ne sont pas sur $C$. Comme les voisins de $v$ forment un ensemble stable, le graphe $G$ est bien composé d'un cycle sur $g$ sommets et les $n-g$ autres sommets sont pendants et tous voisins d'un seul et même sommet du cycle.

- Si $g=n, G$ est un cycle et $\Delta+g=n+2$.

Proposition 4.2. Soit $G$ un graphe connexe d'ordre $n \geq 5$, de degré maximum $\Delta$ et de maille finie $\mathrm{g}$. Alors :

$$
9 \leq \Delta \cdot g \leq\left\lfloor\frac{n+2}{2}\right\rfloor \cdot\left\lceil\frac{n+2}{2}\right\rceil .
$$

De plus la borne inférieure est atteinte si et seulement si le graphe est tel que $\Delta=g=3$, et la borne supérieure pour les graphes ayant l'une des deux structures suivantes :

- un graphe composé d'un cycle de longueur $g=\left\lfloor\frac{n+2}{2}\right\rfloor$ et $\Delta=\left\lceil\frac{n+2}{2}\right\rceil$ sommets pendants tous adjacents au même sommet du cycle;

- un graphe composé d'un cycle de longueur $g=\left\lceil\frac{n+2}{2}\right\rceil$ et $\Delta=\left\lfloor\frac{n+2}{2}\right\rfloor$ sommets pendants tous adjacents au même sommet du cycle.

Preuve. La borne inférieure se démontre exactement comme la borne inférieure de la proposition 4.1.

Pour la borne supérieure et d'après la proposition 4.1, on a :

$$
\Delta \cdot g \leq(n-g+2) \cdot g=f(g) .
$$

Si on considère $f(g)$ comme fonction en $g$, elle atteint son maximum pour $g=$ $\left\lceil\frac{n+2}{2}\right\rceil$ ou $g=\left\lfloor\frac{n+2}{2}\right\rfloor$ avec $f(g)=\left\lfloor\frac{n+2}{2}\right\rfloor \cdot\left\lceil\frac{n+2}{2}\right\rceil$. D'où la borne.

Soit maintenant $G$ un graphe pour lequel la borne supérieure est atteinte. Tout sommet du plus petit cycle de $G$ ne peut avoir que 2 voisins dans ce cycle. Pour que $\Delta=n-g+2$, il faut qu'un sommet de ce cycle soit adjacent à tous les sommets n'appartenant pas à ce cycle. Alors l'ajout de toute arête créerait un cycle de longueur inférieure à $g$. D'où le résultat.

\subsection{LE DEGRÉ MOYEN}

Considérons maintenant le degré moyen et la maille. AGX2 trouve et prouve les bornes suivantes :

$$
2-n \leq \bar{d}-g \leq n-4 \quad \text { et } \quad 2 / n \leq \bar{d} / g \leq(n-1) / 3 .
$$


Les bornes inférieures sont atteintes uniquement pour les cycles, et les bornes supérieures pour les graphes complets.

AGX2 obtient également les conjectures prouvées dans les deux propositions suivantes.

Proposition 4.3. Soit $G$ un graphe connexe d'ordre $n \geq 4$, de degré moyen $\bar{d}$ et de maille finie $g$. Alors :

$$
5 \leq \bar{d}+g \leq n+2
$$

La borne inférieure est atteinte pour les graphes unicycliques dont l'unique cycle est un triangle. La borne supérieure est atteinte pour les graphes complets et les cycles.

Preuve. Pour la borne inférieure : comme $G$ a un cycle, $m \geq n$ et $\bar{d} \geq 2$. Comme $g \geq 3$ la borne s'ensuit. Les conditions $\bar{d}=2$ et $g=3$ impliquent que $G$ est unicyclique et contient un triangle.

La borne supérieure découle de celle de (6) et de $\bar{d} \leq \Delta$. Elle est atteinte pour un graphe régulier. D'après la preuve de la proposition 4.1 , soit $\bar{d}=\Delta=2$ et $g=n$, c'est-à-dire que $G$ est un cycle, soit $\bar{d}=\Delta=n-1$ et $g=3$, c'est-à-dire que $G$ est complet.

Proposition 4.4. Soit $G$ un graphe connexe d'ordre $n \geq 3$, de degré moyen $\bar{d}$ et de maille finie $g$. Alors :

$$
6 \leq \bar{d} \cdot g \leq 3 n-3
$$

La borne inférieure est atteinte pour les graphes unicycliques dont l'unique cycle est un triangle. La borne supérieure est atteinte pour les graphes complets et les cycles.

Preuve. La borne inférieure se démontre comme la borne inférieure de la proposition 4.3.

Pour la borne supérieure, si $g=3$, il est évident que $g \cdot \bar{d} \leq 3 n-3$ avec égalité si et seulement si le graphe est complet. Il nous reste à montrer que si $g>3$, nécessairement $g \cdot \bar{d}<3 n-3$.

Si $g=4$, d'après le théorème de Turan [23], $\bar{d} \leq \frac{n}{2}$, d'où $g \cdot \bar{d} \leq 2 n<3 n-3$.

Si $5 \leq g \leq \frac{n}{2}$, on a, d'après [4], $g \cdot \bar{d} \leq \frac{n^{2}-n g+2 g}{2 n}<3 n-3$ pour tout $n \geq 5$.

Si $\frac{n}{2}<g<n$, on a, d'après [4], $g \cdot \bar{d} \leq(2 n-g-1) \frac{g}{2 n}<3 n-3$.

Si $g=n$, le graphe est un cycle et donc $g \cdot \bar{d}=2 n<3 n-3$ pour tout $n \geq 4$.

\subsection{LE DEGRÉ MINIMUM}

Considérons maintenant le degré minimum et la maille. AGX2 trouve et prouve les résultats suivants :

$$
2-n \leq \delta-g \leq n-4 \quad \text { et } \quad 1 /(n-1) \leq \delta / g \leq(n-1) / 3
$$

La première borne inférieure est atteinte pour les cycles ou pour les graphes composés d'un cycle sur $n-1$ sommets et un sommet pendant, la deuxième est atteinte 
pour les graphes composés d'un cycle sur $n-1$ sommets et un sommet pendant, et les bornes supérieures pour les graphes complets;

$$
\delta+g \geq 4 \quad \text { et } \quad \delta \cdot g \geq 3,
$$

avec égalité si et seulement si $G$ contient un triangle et un sommet pendant. AGX2 obtient également les conjectures prouvées dans les deux propositions suivantes.

Proposition 4.5. Soit $G$ un graphe connexe d'ordre $n \geq 4$, de degré minimum $\delta$ et de maille finie $\mathrm{g}$. Alors :

$$
\delta+g \leq n+2 .
$$

La borne est atteinte pour les graphes complets et les cycles.

Preuve. Le résultat est une conséquence de la proposition 4.1.

Proposition 4.6. Soit $G$ un graphe connexe d'ordre $n \geq 4$, de degré minimum $\delta$ et de maille finie $\mathrm{g}$. Alors :

$$
\delta \cdot g \leq 3 n-3 .
$$

La borne est atteinte pour les graphes complets.

Preuve. Le résultat est une conséquence de la proposition 4.4.

\section{Quelques CONJECTURES OUVERTES}

Nous présentons dans cette dernière section quelques conjectures ouvertes et que nous n'avons, délibérement, pas essayé de prouver. Avant de donner la liste rappelons les définitions suivantes : la distance moyenne $\bar{l}$ d'un graphe $G=(V, E)$ est la moyenne de toutes les distances entre sommets distincts de $G$; l'index (rayon spectral) $\lambda_{1}$ de $G$ est la plus grande valeur propre de la matrice d'adjacence de $G$; l'indice de Randić de $G$ est défini par $R a=\sum_{i j \in E} w_{i j}$, où $w_{i j}=1 / \sqrt{d_{i} d_{j}}, d_{i}$ et $d_{j}$ étant les degrés des sommets de l'arête $i j$; le nombre de domination $\beta$ de $G$ est la cardinalité minimum d'un sous-ensemble de sommets $W$ de $G$ tel que tout sommet de $G$ est soit dans $W$ soit voisin d'un sommet de $W$; le nombre chromatique $\chi$ de $G$ est le nombre minimum de couleurs nécessaires pour colorer les sommets de $G$ de telle sorte que deux sommets adjacents ne soient jamais de la même couleur.

Conjecture 1. Pour tout graphe connexe de maille finie

$$
\frac{\bar{l}}{g} \geq \begin{cases}\frac{n}{4(n-1)} & \text { si } n \text { est pair }, \\ \frac{n+1}{4 n} & \text { si } n \text { est impair }\end{cases}
$$

avec égalité si et seulement si le graphe est un cycle.

Conjecture 2. Pour tout graphe connexe de maille finie

$$
\bar{l} \cdot g \leq \begin{cases}\frac{n^{3}}{4(n-1)} & \text { si } n \text { est pair, } \\ \frac{n^{2}+n}{4} & \text { si } n \text { est impair, }\end{cases}
$$

avec égalité si et seulement si le graphe est un cycle. 
Conjecture 3. Sur l'ensemble des graphes connexes de maille finie et d'ordre $n \geq 4, \lambda_{1}+g$ est minimum pour le graphe composé d'un triangle et d'un chemin incident à ce triangle.

Conjecture 4. Sur l'ensemble des graphes connexes de maille finie et d'ordre $n \geq 4, \lambda_{1} \cdot g$ est minimum pour le graphe composé d'un triangle et d'un chemin incident à ce triangle.

Conjecture 5. Pour tout graphe connexe de maille finie

$$
\lambda_{1} \cdot g \leq 3(n-1)
$$

avec égalité si et seulement si le graphe est un cycle ou un graphe complet.

Il existe des contre-exemples à cette conjecture (trouvés par un rapporteur anonyme). Si $G$ est le graphe constitué d'un cycle $C_{g}$ de longueur $g$ et de $n-g$ sommets pendants tous reliés au même sommet de $C_{g}$, alors l'index de $G$ est supérieur à celui $(\sqrt{n-g+2})$ d'une étoile de degré maximum $n-g+2$. Donc, $\lambda_{1} \cdot g \geq g \cdot \sqrt{n-g+2}$, qui peut être arbitrairement plus grand que $3(n-1)$. Par exemple, pour $g=(n-1) / 2$, et $n \geq 69$.

Conjecture 6. Pour tout graphe connexe de maille finie

$$
R a-g \geq-\frac{n}{2}
$$

avec égalité si et seulement si le graphe est un cycle.

Conjecture 7. Pour tout graphe connexe de maille finie

$$
R a+g \geq \frac{n-3+\sqrt{2}}{\sqrt{n-1}}+\frac{7}{2}
$$

avec égalité si et seulement si le graphe est de taille $m=n$ et contient un sommet dominant.

Conjecture 8. Pour tout graphe connexe de maille finie

$$
R a / g \geq 1 / 2
$$

avec égalité si et seulement si le graphe est un cycle.

Conjecture 9. Pour tout graphe connexe de maille finie

$$
R a \cdot g \geq \frac{3 n-9+3 \sqrt{2}}{\sqrt{n-1}}+\frac{1}{2}
$$

avec égalité si et seulement si le graphe est de taille $m=n$ et contient un sommet dominant. 
Conjecture 10. Pour tout graphe connexe de maille finie

$$
\beta-g \geq-\left\lfloor\frac{2 n}{3}\right\rfloor .
$$

La borne est atteinte pour toute valeur de $n$ (par le cycle qui n'est toutefois pas unique).

Conjecture 11. Pour tout graphe connexe de maille finie

$$
\beta / g \geq 1 / 3
$$

avec égalité si et seulement si le graphe contient un sommet dominant et un triangle.

Conjecture 12. Pour tout graphe connexe de maille finie

$$
\chi \cdot g \leq 3 n
$$

avec égalité si et seulement si le graphe est un cycle impair ou un graphe complet.

Cette conjecture a été prouvée par un des rapporteurs anonymes de cet article.

Preuve. Comme la maille est finie, le graphe contient un cycle, et aussi $\chi \geq 2$. Si $\chi=2$, alors $\chi \cdot g \leq 2 n$.

Supposons que $\chi \geq 3$ et soit $V_{1}, V_{2}, \ldots, V_{\chi}$ les classes de couleurs du graphe. Notons $H$ le sous-graphe induit par la réunion des trois plus petites classes de couleurs. Alors $H$ contient au plus $3 n / \chi$ sommets. (En effet, soit $k$ le nombre minimum de sommets dans un triplet de classes de couleurs. En faisant la somme des sommets sur l'ensemble des triplets de classes, chaque sommet est compté $\left(\begin{array}{c}\chi-1 \\ 2\end{array}\right)$ fois, donc $k \cdot\left(\begin{array}{l}\chi \\ 3\end{array}\right) \leq n \cdot\left(\begin{array}{c}\chi-1 \\ 2\end{array}\right)$, qui donne $k \leq 3 n / \chi$.) De plus, $H$ ne peut pas être biparti, sinon le graphe d'origine serait coloriable avec $\chi-1$ couleurs. Par conséquent, $H$ contient un cycle impair, dont la longueur est au plus $3 n / \chi$, et donc $g \leq 3 n / \chi$.

Si $\chi=3$, on a l'égalité si et seulement si $H$, et par conséquent le graphe, est isomorphe à un cycle impair.

Si $g=3$, on a l'égalité si et seulement si $\chi=n$, c'est-à-dire que le graphe est complet.

Supposons que $g>3$ et $\chi>3$ et que la borne est atteinte. Alors $H$ est isomorphe à un cycle impair $C_{g}$, donc $g$ est impair et alors $g \geq 5$. Considérons une autre classe de couleurs $V_{i}$ non incluse dans $H . V_{i}$ contient un sommet $u$ adjacent à un sommet de chacune des autres classes, sinon les sommets de $V_{i}$ peuvent être répartis sur les autres classes et avoir une coloration avec $\chi-1$ couleurs. Le sous-graphe induit par $H$ et $u$ est une roue, avec au moins 3 rayons. Sa maille est au plus $2+g / 3$, qui est inférieure à $g$, ce qui est une contradiction.

Alors, la borne est atteinte si et seulement si le graphe est un cycle impair ou un graphe complet. 


\section{Conclusion}

La comparaison de $g$ avec $\alpha, r, D, \Delta, \bar{d}$ et $\delta$ implique 48 bornes inférieures ou supérieures. Dans tous les cas, AGX2 a obtenu des résultats complets, c'est-à-dire des bornes les meilleures possibles pour tout $n$ ainsi qu'une caractérisation des graphes extrêmes. De plus, 22 de ces bornes ont été prouvées automatiquement par AGX2 et les 26 autres à la main.

Ces résultats complets sont dus à la relative facilité de manipulation des invariants considérés. Il ne semble pas en aller de même pour les 12 autres conjectures mentionnées, qui font usage d'invariants moins commodes comme $\lambda_{1}$ ou $R$. Ceci est illustré par la relative difficulté de la preuve de la conjecture 12 donnée par un rapporteur anonyme.

Remerciements. Les auteurs remercient Gilles Caporossi pour des discussions concernant le système AGX2 et les conjectures obtenues. Ils remercient également un rapporteur anonyme pour de nombreuses suggestions qui ont permis d'améliorer la présentation de l'article, ainsi que pour une réfutation de la conjecture 5 et une preuve de la conjecture 12.

\section{RÉFÉRENCES}

[1] M. Aouchiche, J.-M. Bonnefoy, A. Fidahoussen, G. Caporossi, P. Hansen, L. Hiesse, J. Lacheré and A. Monhait, Variable Neighborhood Search for Extremal Graphs. 14. The AutoGraphiX 2 System. Global Optimization : From Theory to Implementation, edited by L. Liberti and N. Maculan, Springer (2005).

[2] M. Aouchiche, G. Caporossi and P. Hansen, Automated Comparison of Graph Invariants. Les Cahiers du GERAD, G-2005-40, rapport technique, HEC Montréal (2005) 21 pages.

[3] S. Belhaiza, N.M.M. de Abreu, P. Hansen and C.S. Oliveira, Variable Neighborhood Search for Extremal Graphs 11. Bounds on Algebraic Connectivity, edited by D. Avis, A. Hertz and O. Marcotte, Graph Theory and Combinatorial Optimization, Dordrecht, Kluwer (2005) $1-16$.

[4] R. C. Brigham and R. D. Dutton, A Compilation of Relations between Graph Invariants. Networks 21 (1991) 421-455.

[5] G. Caporossi, D. Cvetkovic, I. Gutman and P. Hansen, Variable Neighborhood Search for Extremal Graphs. 2. Finding Graphs with Extremal Energy. J. Chem. Inform. Comput. Sci. 39 (1999) 984-996.

[6] G. Caporossi, I. Gutman and P. Hansen, Variable Neighborhood Search for Extremal Graphs. 4. Chemical Trees with Extremal Connectivity Index. Comput. Chem. 23 (1999) 469-477.

[7] G. Caporossi and P. Hansen, Variable Neighborhood Search for Extremal Graphs. I. The AutoGraphiX System. Discrete Math. 212 (2000) 29-44.

[8] G. Caporossi and P. Hansen, Variable Neighborhood Search for Extremal Graphs. V. Three Ways to Automate Finding Conjectures. Discrete Math. 276 (2004) 81-94.

[9] F.R.K. Chung, The Average Distance and the Independence Number. J. Graph Theory 12 (1988) 229-235.

[10] D. Cvetković, S. Simić, G. Caporossi and P. Hansen, Variable Neighborhood Search for Extremal Graphs. III. On the Largest Eigenvalue of Color-Constrained Trees. Linear Multilinear Algebra 49 (2001) 143-160.

[11] D. Cvetković and S. Simić, Graph Theoretical Results Obtained by the Support of the Expert System "GRAPH" - an Extended Survey. In [13]. 
[12] S. Fajtlowicz, On Conjectures of Graffiti. Discrete Math. 72 (1988) 113-118.

[13] Graphs and Discovery. DIMACS Series in Discrete Math. and Theoretical Computer Science, edited by S. Fajtlowicz, P. Fowler, P. Hansen, M. Janowitz and F. Roberts, Providence, AMS (2005).

[14] I. Gutman, P. Hansen and H. Mélot, Variable Neighborhood Search for Extremal Graphs. 10. Comparison of Irregularity Indices for Chemical Trees. J. Chem. Inform. Comput. Sci. (2005, to appear).

[15] P. Hansen, Computers in Graph Theory. Graph Theory Notes of New York 43 (2002) 20-34.

[16] P. Hansen, How Far Is, Should and Could Be Conjecture-Making in Graph Theory an Automated Process? In [13].

[17] P. Hansen and H. Mélot, Variable Neighborhood Search for Extremal Graphs. 6. Analyzing Bounds for the Connectivity Index. J. Chem. Inform. Comput. Sci. 43 (2003) 1-14.

[18] P. Hansen and H. Mélot, Variable Neighborhood Search for Extremal Graphs. 9. Bounding the Irregularity of a Graph. In [13].

[19] P. Hansen and N. Mladenović, Variable Neighborhood Search : Principles and Applications. Eur. J. Oper. Res. 130 (2001) 449-467.

[20] N. Mladenović and P. Hansen, Variable Neighborhood Search. Comput. Oper. Res. 24 (1997) $1097-1100$.

[21] E.A. Nordhaus and J.W. Gaddum, On Complementary Graphs. Amer. Math. Monthly 63 (1956) $175-177$.

[22] B.A. Smith, Private communication (2004).

[23] P. Turán, Eine Extremalaufgabe aus der Graphentheorie. (Hungarian) Mat. Fiz. Lapok 48 (1941) 436-452.

[24] Written on the wall. Electronic file available from http ://math.uh.edu/ clarson/ (1999). 